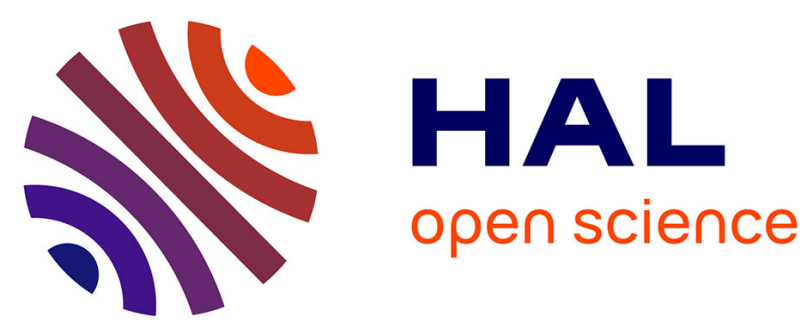

\title{
Titanium-based silicide quantum dot superlattices for thermoelectrics applications
}

Guillaume Savelli, Sergio Silveira Stein, Guillaume Bernard-Granger, Pascal Faucherand, Laurent Montès, Stefan Dilhaire, Gilles Pernot

\section{To cite this version:}

Guillaume Savelli, Sergio Silveira Stein, Guillaume Bernard-Granger, Pascal Faucherand, Laurent Montès, et al.. Titanium-based silicide quantum dot superlattices for thermoelectrics applications. Nanotechnology, 2015, 26 (27), pp.275605. 10.1088/0957-4484/26/27/275605 . hal-01407030

\section{HAL Id: hal-01407030 \\ https://hal.science/hal-01407030}

Submitted on 1 Dec 2016

HAL is a multi-disciplinary open access archive for the deposit and dissemination of scientific research documents, whether they are published or not. The documents may come from teaching and research institutions in France or abroad, or from public or private research centers.
L'archive ouverte pluridisciplinaire HAL, est destinée au dépôt et à la diffusion de documents scientifiques de niveau recherche, publiés ou non, émanant des établissements d'enseignement et de recherche français ou étrangers, des laboratoires publics ou privés.

\section{다(1)(2)}

Distributed under a Creative Commons Attribution - ShareAlikel 4.0 International 
Titanium-based silicide quantum dot superlattices for thermoelectrics applications

This content has been downloaded from IOPscience. Please scroll down to see the full text. 2015 Nanotechnology 26275605

(http://iops ienc .iop.org/0957-4484/26/27/275605)

View the table of contents for this issue, or go to the journal homepage for more

Download details:

IP Address: 147.210.24.83

This content was downloaded on 18/11/2016 at 09:13

Please note that terms and conditions apply.

You may also be interested in:

Significant enhancement of the thermoelectric figure of merit of polycrystalline Si films by reducing grain size

K Valalaki, N Vouroutzis and A G Nassiopoulou

Half-Heusler thermoelectrics: a complex class of materials

Jan-Willem G Bos and Ruth A Downie

Thermal conductivity of isotopically controlled silicon nanostructures

$\mathrm{H}$ Bracht, S Eon, R Frieling et al.

Tuning phonon properties in thermoelectric materials

G P Srivastava

Large-area thermoelectric high-aspect-ratio nanostructures by atomic layer deposition Mikko Ruoho, Taneli Juntunen and Ilkka Tittonen

Concepts for medium-high to high temperature thermoelectric heat-to-electricity conversion: a review of selected materials and basic considerations of module design

Gabi Schierning, Ruben Chavez, Roland Schmechel et al. 


\title{
Titanium-based silicide quantum dot superlattices for thermoelectrics applications
}

\author{
Guillaume Savelli ${ }^{1}$, Sergio Silveira Stein ${ }^{1}$, Guillaume Bernard-Granger ${ }^{1}$, \\ Pascal Faucherand ${ }^{1}$, Laurent Montès ${ }^{2}$, Stefan Dilhaire ${ }^{3}$ and Gilles Pernot ${ }^{3}$ \\ ${ }^{1}$ CEA, Liten, Thermoelectricity Laboratory, 17 rue des Martyrs, 38000 Grenoble, France \\ ${ }^{2}$ IMEP LAHC Laboratory, 3 Parvis Louis Néel, 38000 Grenoble, France \\ ${ }^{3}$ LOMA, 351 cours de la Libération, 33405 Talence, France \\ E mail: guillaume.savelli@cea.fr
}

\begin{abstract}
Ti-based silicide quantum dot superlattices (QDSLs) are grown by reduced-pressure chemical vapor deposition. They are made of titanium-based silicide nanodots scattered in an $n$-doped SiGe matrix. This is the first time that such nanostructured materials have been grown in both monocrystalline and polycrystalline QDSLs. We studied their crystallographic structures and chemical properties, as well as the size and the density of the quantum dots. The thermoelectric properties of the QDSLs are measured and compared to equivalent SiGe thin films to evaluate the influence of the nanodots. Our studies revealed an increase in their thermoelectric properties

specifically, up to a trifold increase in the power factor, with a decrease in the thermal conductivity making them very good candidates for further thermoelectric applications in cooling or energy-harvesting fields.
\end{abstract}

\section{Introduction}

Thermoelectricity is one of the most promising research fields for future applications of clean energy generation. Thermoelectric systems can convert thermal energy into electrical energy for energy-harvesting applications (the Seebeck effect), or reciprocally for cooling applications (the Peltier effect). Thermoelectric materials are evaluated by their figure of merit, ZT, which is defined in equation (1), where $S$ is the Seebeck coefficient, $\lambda$ is the thermal conductivity, $\rho$ is the electrical resistivity, and $\mathrm{T}$ is the temperature.

$$
Z T=\left(S^{2} \times T\right) /(\rho \times \lambda)
$$

A high ZT value directly captures the efficiency of a material used as a thermoelectric device.

Two of the most widespread thin film materials currently used are $\mathrm{Bi}_{2} \mathrm{Te}_{3}$ and $\mathrm{PbTe}$, both of which include rare or toxic elements [1, 2].
An environmentally clean alternative to these materials is a doped SiGe film with low concentrations of Ge. This material has several advantages such as being abundant, relatively low in cost, well studied, and compatible with the microelectronics industry, but it exhibits poor thermoelectric performance at room temperature.

Among the different strategies used to improve the figure of merit of the materials, one of the most encouraging is the use of nanostructure engineering [3].

Nanostructuration of materials could improve ZT materials by increasing the power factor (defined as $S^{2} / \rho$ ) and/or reducing the thermal conductivity.

At the short scale, SiGe-based nanostructured materials have already shown a significant decrease in thermal conductivity compared to similar $\mathrm{SiGe}$ thin films, $\mathrm{Si} / \mathrm{SiGe}$ superlattices, and $\mathrm{Si}_{x} \mathrm{Ge}_{1-x} / \mathrm{Si}_{y} \mathrm{Ge}_{1-y}$ quantum dot superlattices (QDSLs) [4 6]. This is mainly due to a reduction of the crystal's lattice thermal conductivity, which is obtained by 


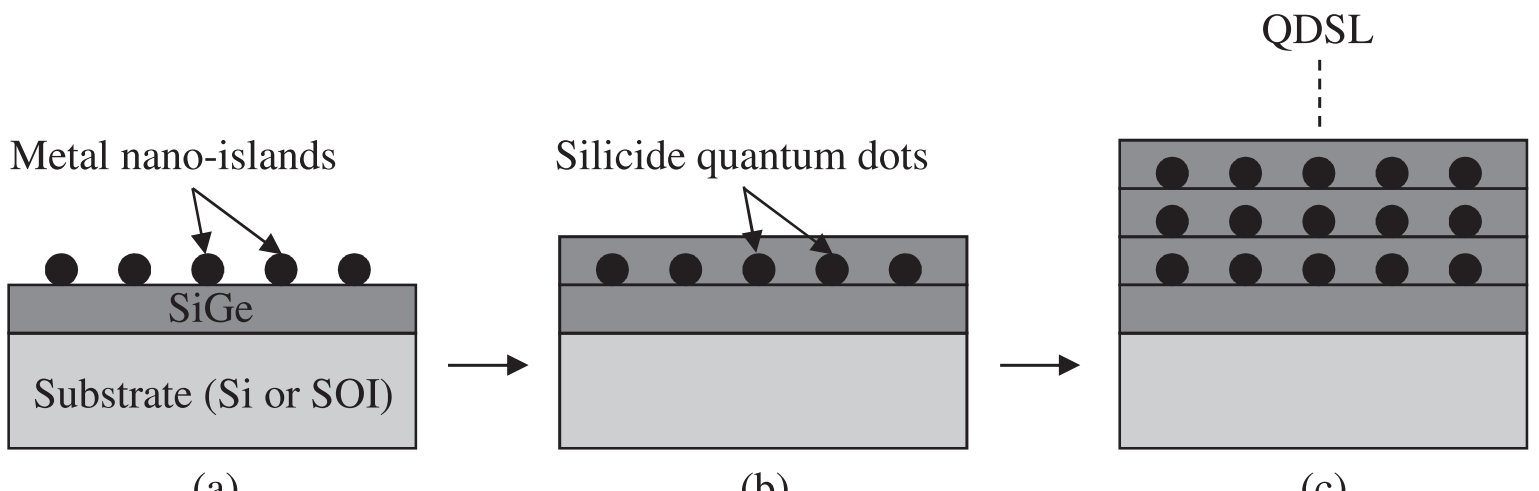

(a)

(b)

(c)

Figure 1. Schematic drawing of the successive steps to grow a QDSL by CVD. (a) Metal nanoislands are grown on a SiGe thin film. (b) A $\mathrm{SiGe}$ layer is deposited to embed the islands and form the QDs. Steps (a) and (b) are repeated to produce a QDSL, represented in (c).

adding phonon diffusion mechanisms as layer interfaces and nanodots.

It has recently been shown that the inclusion of metallic silicide nanodots (like $\mathrm{TiSi}_{2}$ ) in a $\mathrm{SiGe}$ matrix could theoretically lead to an up to fourfold reduction of the material's thermal conductivity without altering its electrical conductivity or its Seebeck coefficient [7]. In their study, these authors proposed silicide dots with diameters and thicknesses in the $550 \mathrm{~nm}$ and $4050 \mathrm{~nm}$ ranges, respectively, with a $3 \%$ quantum dot (QD) density. This result was recently experimentally demonstrated at the bulk scale, but it has never been demonstrated at the thin film scale [8] because these nanostructured thin film materials have never been grown or presented in the literature.

In this paper, we present the first instance of the growth of QDSL thin films based on titanium silicides inside doped SiGe mono- and polycrystalline matrixes, as well as a complete study of their chemical structures and thermoelectric (TE) properties.

\section{QDSL growth mechanism}

This section describes the QDSL growth mechanism, which is illustrated in figure 1. The deposition of QDSL by reducedpressure chemical vapor deposition (CVD) can be divided into three steps. The first step is the deposition of $\mathrm{Ti}$ nanoislands onto a SiGe thin film. In the second step, the nanoislands are embedded by the deposition of a SiGe thin film, simultaneously forming QDs by the reaction between the $\mathrm{Ti}$ atoms and the $\mathrm{Si}$ atoms. Finally, these steps are repeated to produce a QDSL.

In this paper, we differentiated the expression 'nanoisland' from the term 'QD', in the sense that the former is related to the first steps of the growth, where the deposited $\mathrm{Ti}$ atoms form quasiplanar nanometric structures. Once they react with the Si atoms and form silicides, spherical crystalline structures called QDs are also formed.

All QDSL and reference samples are grown in a modified Epi-Centura reduced-pressure CVD industrial cluster tool from Applied Materials. Two different substrates are used. The polycrystalline samples are grown on $100 \mathrm{~mm}$, lightly doped $\mathrm{Si}$ wafers where a $200 \mathrm{~nm} \mathrm{SiO}_{2}$ insulating layer was previously grown by plasma-enhanced CVD. The monocrystalline samples are epitaxially grown on lightly doped silicon on insulator (SOI) wafers.

This technique has already been used to successfully grow nanostructured silicon-based thin films [4, 13, 14].

The precursor chosen for the Ti-based QD growth is $\mathrm{TiCl}_{4}$, which is liquid at ambient temperatures. A specific, adapted apparatus allowing the controlled recovery of the precursor vapor was built and coupled to the Centura tool. This apparatus has a temperature and pressure control system, and $\mathrm{H}_{2}$ is employed as the carrier gas to send the gaseous portion of the precursor to the reaction chamber.

The SiGe layers are grown in the same chamber using $\mathrm{SiH}_{4}$ and $\mathrm{GeH}_{4}$ as precursors. The samples were doped in situ using $\mathrm{PH}_{3}$ gas. The Ge content of the QDSL SiGe layers is fixed at around $13 \%$. As shown in figure 1, to create a QDSL, a doped $\mathrm{SiGe}$ layer is first grown on the substrate. After that, the $\mathrm{SiH}_{4}, \mathrm{GeH}_{4}$, and $\mathrm{PH}_{3}$ gas flows are stopped and the $\mathrm{TiCl}_{4}$ rich $\mathrm{H}_{2}$ is sent to the chamber for different periods of time to produce the Ti-based QDs. Then, another doped SiGe layer is grown to embed the nanoparticles, and so on. Deposition temperature and pressure are $800{ }^{\circ} \mathrm{C}$ and $10 \mathrm{Torr}$, respectively. The total thickness of the QDSL and the reference sample is $1 \mu \mathrm{m}$. The QDSLs are composed of 16 alternating QD and SiGe layers. A $200 \mathrm{~nm} \mathrm{SiGe} \mathrm{buffer} \mathrm{layer} \mathrm{is} \mathrm{deposited}$ before the QDSL. This is done to reduce potential dislocations due to the lattice mismatch between the SiGe thin films and the Si substrate, to lower the interdiffusion between the $\mathrm{Ge}$ atoms from the QDSL and the Si atoms from the substrate, and to maintain a constant $\mathrm{Ge}$ content trough in all $\mathrm{SiGe}$ layers of the QDSL.

\section{QDSL structural analyses}

The QDSL structures are analyzed by transmission electronic microcopy (TEM). These TEM analyses are performed at $200 \mathrm{kV}$, including high-angle annular dark field (HAADF) images in the scanning mode (STEM), which allow us to acquire both high-resolution images and chemical mapping using the energy-dispersive $\mathrm{x}$-ray spectroscopy (EDS) 


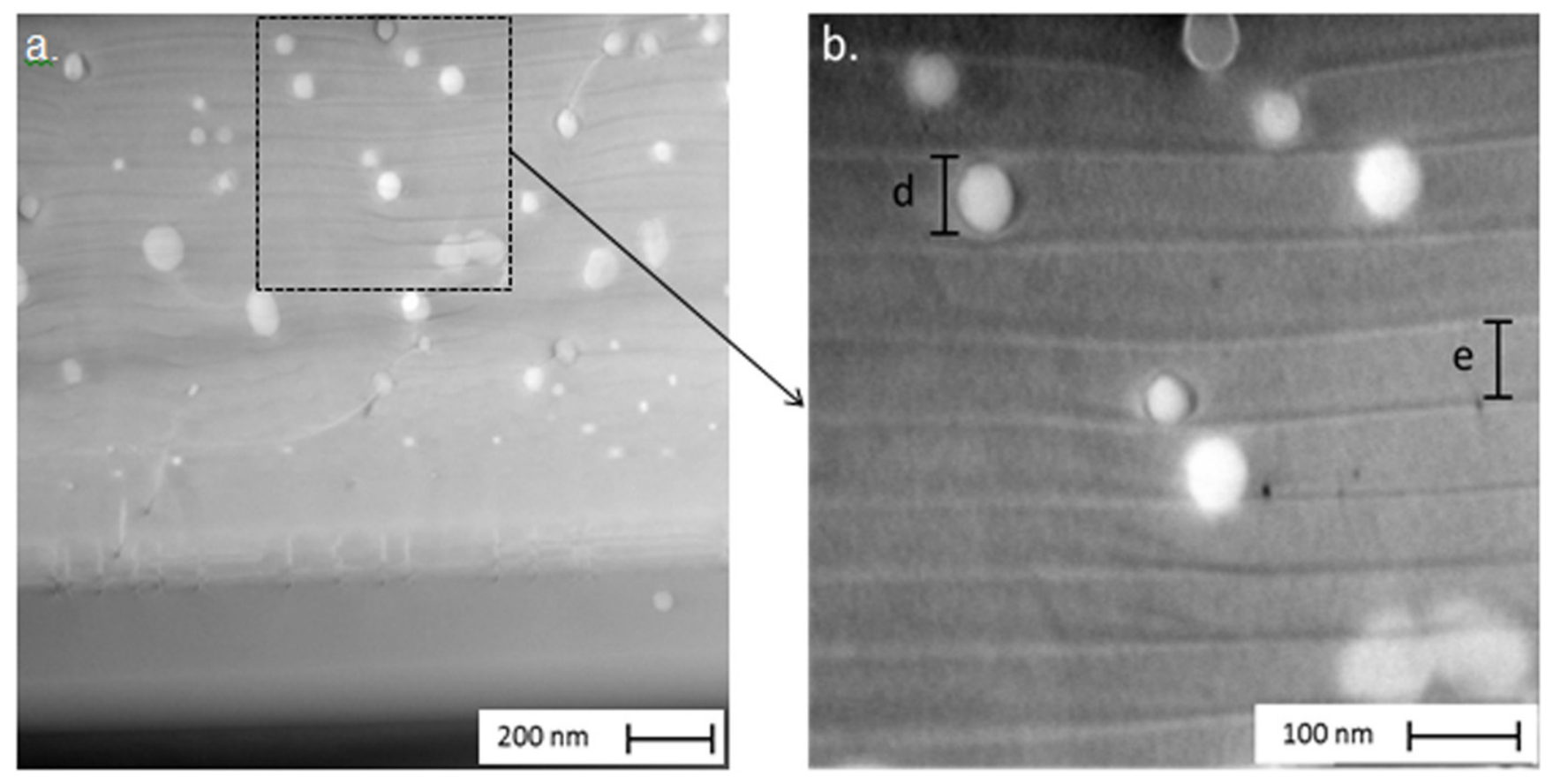

Figure 2. Cross sectional STEM/HAADF images of the monocrystalline sample. (a) A global view of the QDSL showing the Ti based QD, and (b) a higher magnification showing a QD with diameter, $d=66 \mathrm{~nm}$, and layer thickness, $e=65 \mathrm{~nm}$.

technique. The samples are prepared using a tripod polishing technique. These features allow us to acquire three types of images. The first one consists of global cross-plane views of the QDSL, which permit us to measure the QDs' diameters, each layer's thickness, and the total thickness of the samples. The second mode used involves high-resolution images, which allow us to analyze individual QDs and the distance between crystallographic planes, and to determine the phase of the particle. The third class of TEM consists of the chemical mappings, which are obtained with the EDS technique coupled with the STEM mode. This technique is based on $x$-ray emissions due to the relaxation of excited electrons, which assume discrete and particular energy values for each element of the periodic table. The obtained chemical mapping produces high-spatial-resolution images of the position of the different elements forming the material, estimates the $\mathrm{Ge}$ content of each samples, and confirms the phase of the QDs.

The results of these analyses are presented for both mono- and polycrystalline QDSLs. At the end of this section, we compare the results and the growth of each sample.

\subsection{Monocrystalline sample}

The overall view of the monocrystalline sample is presented in figure 2. An initial observation is that the particles of the lower layers appear to be smaller than the particles of the upper layers. This occurs because of the high mobility of the dots inside the SiGe matrix. When we embed the nanoislands with the successive SiGe monolayers (from step (a) to step (b) in figure 1), a large percentage of the particles migrates to the surface of the sample. Thus, during the stacking of the layers, the same mechanism occurs, but instead of migrating up to the surface, the QDs coalesce inside the material, resulting in particles with larger diameters.

Surface images of the QDSL show no QDs on the surface, so one can conclude that when the QDs reach a critical diameter, their mobility inside the SiGe matrix is lowered and they remain inside the material. In figure 2(b), the QD diameter, d, and the layer thickness, e, are measured. A statistical measurement of this image provides the mean values of $d=66 \mathrm{~nm}, e=70 \mathrm{~nm}$, and a 2.3\% QD density, which are close to the theoretical optimized values (see section 1).

Figure 3 presents a high-resolution image of a single QD inside the monocrystalline matrix. The measure of the plane distances result from $d_{\mathrm{p}} \approx 8.21 \AA$, which corresponds to the (100) $\mathrm{TiSi}_{2}$ planes.

The EDS chemical mapping of the sample is presented in figure 4. Two main observations can be deduced from these images. First, looking at the Ti signal in figure 4(d), one can easily see that the QDs are rich in Ti atoms, confirming the measurements discussed above. The second important piece of information is obtained by analyzing the dopant (phosphorus) and the signal (figure 4(e)). Due to a chemical affinity between the two materials, an accumulation of phosphorus is observed in the zones corresponding to the QD. A similar result was observed in [8]. In this reference, however, the inclusions were Mo-based QDs. A punctual EDS analysis inside the $\mathrm{SiGe}$ embedding layer resulted in a Ge content of $12.7 \%$ (atomic fraction). The same analysis was performed inside a QD where a Ti content of $31 \%$ was obtained, confirming that the QD phase is $\mathrm{TiSi}_{2}$. Comparing figures 4(a), (d), and (e), a dopant accumulation near the QD is observed. This accumulation, which was previously reported in the literature for bulk phosphorous-doped SiGe samples with Mobased inclusions [8], stems from the high affinity between the 


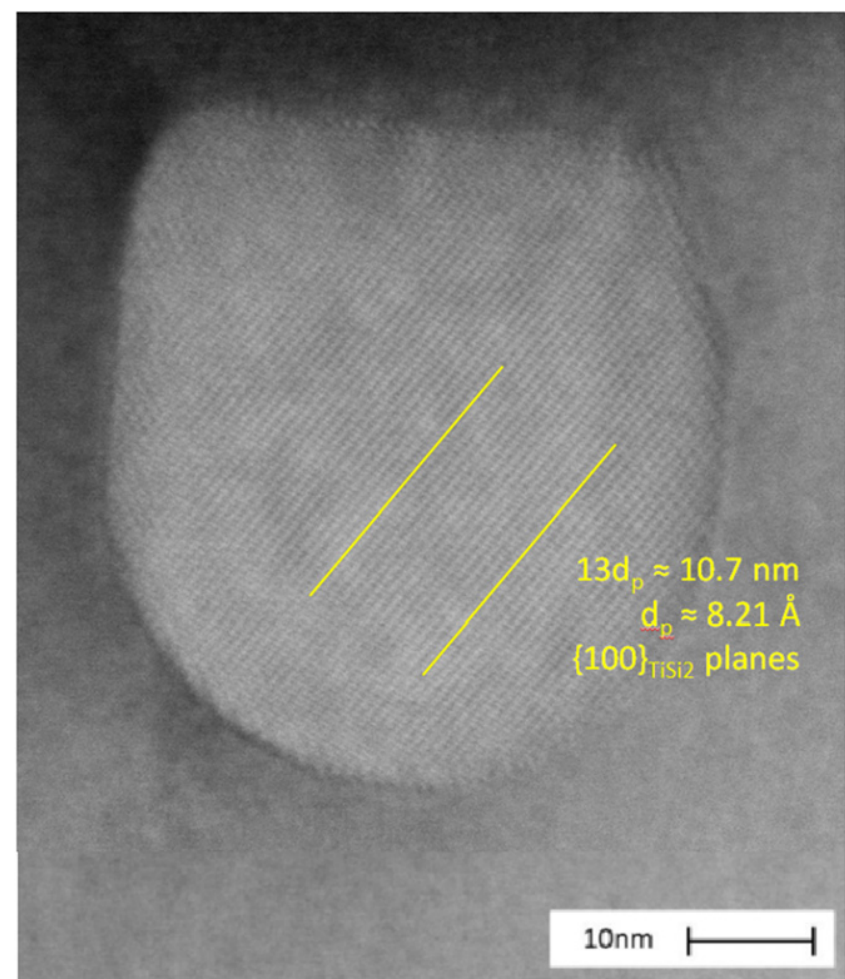

Figure 3. High resolution STEM/HAADF image of a QD inside the monocrystalline sample. The plane distances correspond to the (100) planes of the $\mathrm{TiSi}_{2}$ phase.

metal silicide and the dopant atoms, which causes a precipitation around the QD.

It has also been shown that this effect has a huge impact on the material's power factor [9]. The authors observed an increase in the material's electrical conductivity due to an increase in the carriers' mobility, without any changes in the Seebeck coefficient. This effect, called modulation doping, is obtained by spatially separating carriers from their parent atoms.

\subsection{Polycrystalline sample}

Considering the polycrystalline n-doped Ti-based QDSL, a global cross-sectional TEM image is presented in figure 5. Comparing the two distinct zones, ' 1 ' and '2', in figure 5(a), there is a difference in the QD density between the two zones, with zone 2 being richer in QDs than zone 1. Zone 2 corresponds to an individual grain, and the particles tend to agglomerate in the grain boundaries. The underlying reason for the preferential growth near the grain boundaries is that these highly disordered sites lower the energy for the nucleation of the Ti phase. By statistically measuring the particle diameter and the thickness of the layers of this sample, a mean QD diameter of $d=42 \mathrm{~nm}$, a thickness of $e=54 \mathrm{~nm}$, and a QD density of $2.7 \%$ are obtained. Those values are in good agreement with the theoretical optimized values (see section 1).

As previously stated, one can see that the particles of the lower layers corresponding to the first stages of the QDSL growth are smaller than the upper QDs. These two features indicate that a coalescence effect occurs in the polycrystalline sample during the growth, but at a smaller scale than in the monocrystalline sample. The mean diameter in this case $(42 \mathrm{~nm})$ is smaller than that of the monocrystalline QDSL $(66 \mathrm{~nm})$. As a result, the grain boundaries act as a barrier for the diffusion of the QDs inside the matrix, reducing the coalescence effect.

Figure 6 presents a high-resolution image of an individual QD inside the polycrystalline sample. In this image, two sets of planes are identified, corresponding to the distances of the (110) and (210) planes of the $\mathrm{Ti}_{3} \mathrm{Si}$ tetragonal phase. A different phase is obtained when a polycrystalline material is grown. In this case, the obtained phase $\left(\mathrm{Ti}_{3} \mathrm{Si}\right)$ is much richer in $\mathrm{Ti}$ than the phase $\left(\mathrm{TiSi}_{2}\right)$ of the monocrystalline sample, suggesting that the reaction between $\mathrm{Si}$ and $\mathrm{Ti}$ is slower.

EDS chemical mapping of this sample is presented in figure 7. The information obtained from these analyses is very similar to the information obtained for the monocrystalline sample (i.e., the good correspondence between the quantum dots, the $\mathrm{Ti}$, and the $\mathrm{P}$ dopant signals). Quantitative EDS analysis of the matrix provides a Ge content of $12 \%$, which is similar to the monocrystalline sample. By performing a quantitative analysis of a single QD, a Ti content of 70\% was obtained, which is close to the value corresponding to the $\mathrm{Ti}_{3} \mathrm{Si}$ phase $(\mathrm{Ti}=75 \%)$.

Finally, by comparing figures 7(a), (d), and (e), the same dopant accumulation near the QD is observed, which also proves a modulation doping effect.

\section{QDSL thermoelectric characterizations}

In this section, we present the measurement of the QDSL thermoelectric power factor and thermal conductivity.

\subsection{Characterization methods}

The thermoelectric power factor is usually defined as the product of the electrical conductivity and the Seebeck coefficient square. An ULVAC ZEM-3 device is used to measure both parameters at temperatures ranging from room temperature to $200^{\circ} \mathrm{C}$.

Usually this device is used to measure the TE properties of bulk materials. For thin-film samples, one must adapt their structures to be compatible with this method. A schematic representation of the system is shown in figure 8 .

As a first step, we evaporate two rectangular strips of $\mathrm{Al} /$ $\mathrm{Au}$ onto the thin film's surface. The $200 \mathrm{~nm}$ Al metal deposition by evaporation is chosen to provide good ohmic contact with the SiGe-based material. An additional $100 \mathrm{~nm}$ thick Au layer is deposited using the same equipment to protect the surface against oxidation.

Then, the sample is mounted between the electrodes (figure 8(a)). The upper electrode serves as a heating element. A polyimide electrical insulating film is inserted between the sample edge and the electrode, and a copper wire is positioned to electrically connect the electrodes and the $\mathrm{Al} / \mathrm{Au}$ strips on the sample's surface. The Si (or SOI) substrate does 

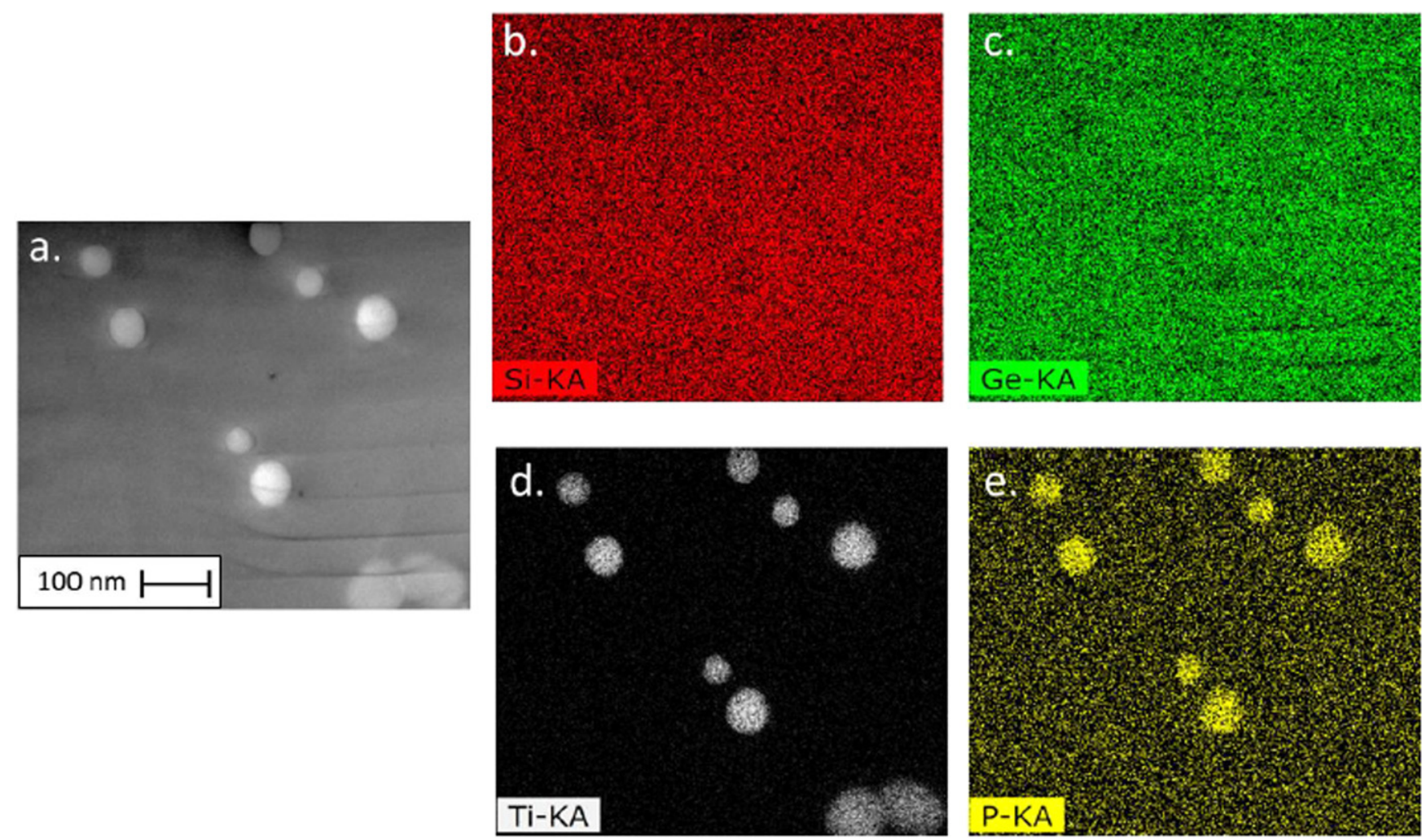

Figure 4. EDS chemical mapping of the monocrystalline sample: (a) STEM/HAADF image, (b) silicon signal, (c) germanium signal, (d) titanium signal, and (e) phosphorus signal. Note the similarity between the QDs, the titanium, and the phosphorus signals.

not interfere with the electrical measurements, and the electrical contacts are established only between the electrodes and the thin film.

The equipment works simultaneously in two modes. The first mode measures the electrical conductivity, and the second measures the Seebeck coefficient. The first mode is represented in figure $8(\mathrm{~b})$ and can be compared to a four-point probe method. An electrical current, I, is imposed through the sample and the resulting voltage, $\mathrm{V}$, is measured using the thermocouples as electrodes. Using Ohm's law, the thin film's electrical resistance is obtained. The electrical conductivity is then calculated using the sample length 1 , the width $\mathrm{w}$, and the thin film's thickness e, according to equation (2):

$$
\sigma=(l \times I) /(w \times e \times V) .
$$

The Seebeck coefficient-measurement setup is presented in figure 8(c). The thermocouples in the center of the sample are used to measure the temperature difference, $\Delta T$. Then the driver software switches the electrode mode to measure the voltage created by the temperature gradient. The carriers' concentration and mobility are measured using a Hall effect method.

Note that these techniques measure the properties along the in-plane direction (i.e., parallel to the thin film's surface).

The thermal conductivity of the samples was measured at room temperature by time-domain thermoreflectance (TDTR). This optical technique consists of exciting the surface of the samples with a femtosecond laser pulse, creating a transient heat source. Another laser pulse monitors the variation of the sample's reflectivity. Prior to any measurement, the samples are capped with a $70 \mathrm{~nm}$ aluminum thin film that acts as a transducer. Then, by using a three-dimensional heat-diffusion model, it is possible to fit the experimental data and extract the material's thermal conductivity [10, 11]. Unlike the electrical conductivity and the power factor, the TDTR technique measures the cross-plane thermal conductivity component of thin films.

Note that an SiGe reference sample has been grown with the same Ge content (13\%) and electrical conductivity as the corresponding mono- and polycrystalline QDSLs. These references are made to compare and evaluate the role of the QDs' inclusion on the material's Seebeck coefficient and thermal conductivity.

\subsection{Thermoelectric parameters}

The evolution of the Seebeck coefficient, electrical resistivity, and power factor of the QDSLs and their corresponding SiGe reference samples as a function of the temperature are presented in figures 9 and 10 for the monocrystalline and polycrystalline structures, respectively. Table 1 presents the carriers' concentration, $n$, mobility, $\mu$, and thermal conductivity, $\lambda$, of the monocrystalline and polycrystalline QDSLs and their SiGe references at room temperature. In figures 9(a) and 10(a), the behavior of the electrical resistivity is, as expected, the same for the QDSL and the reference sample. 

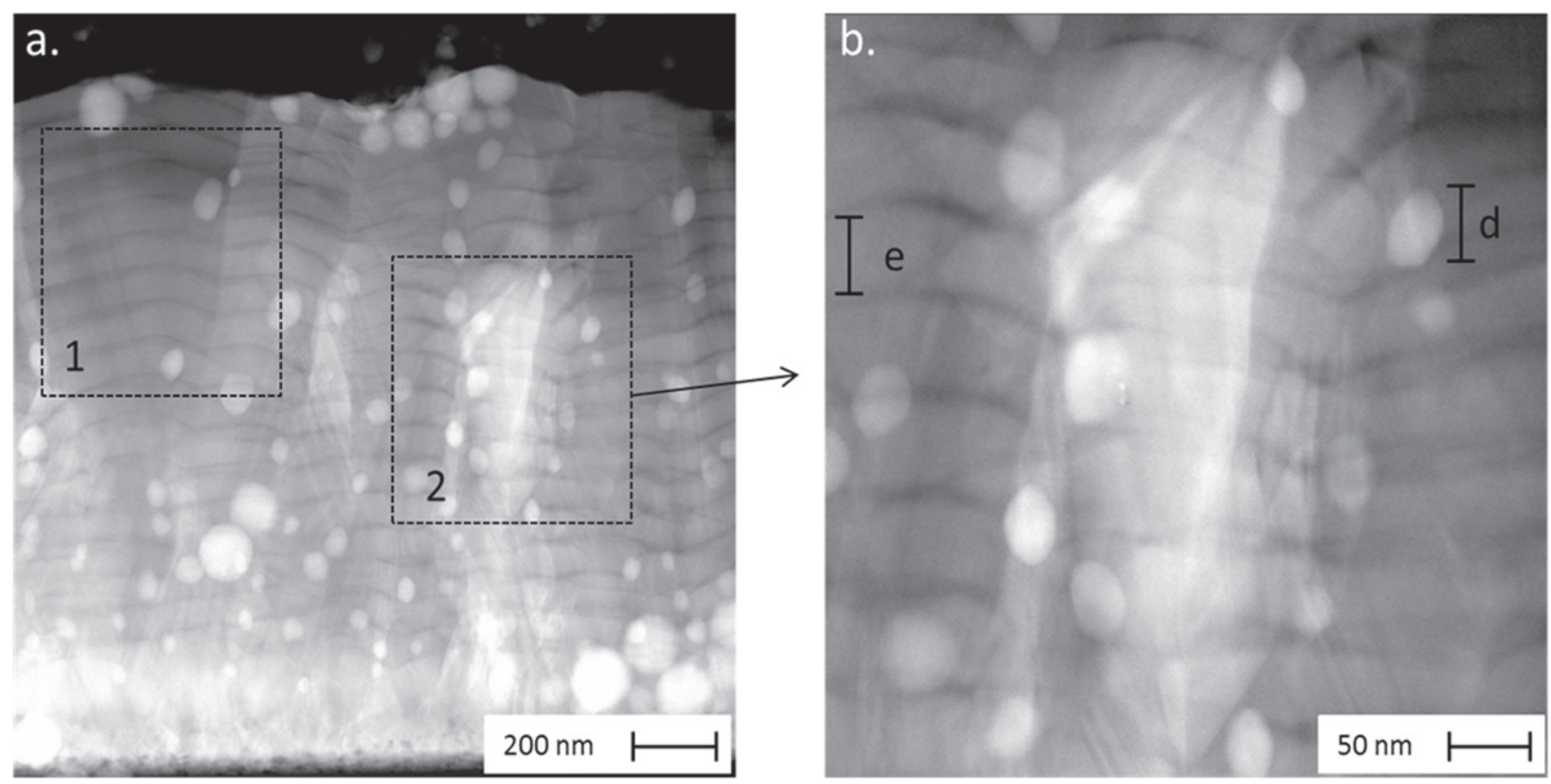

Figure 5. Cross sectional STEM/HAADF image of the polycrystalline sample. (a) A global view of the QDSL, showing the Ti based QD and zones 1 and 2 with different QD densities. (b) A higher magnification of the image, showing a SiGe grain and a QD with diameter, $d=44 \mathrm{~nm}$, and layer thickness, $e=50 \mathrm{~nm}$.

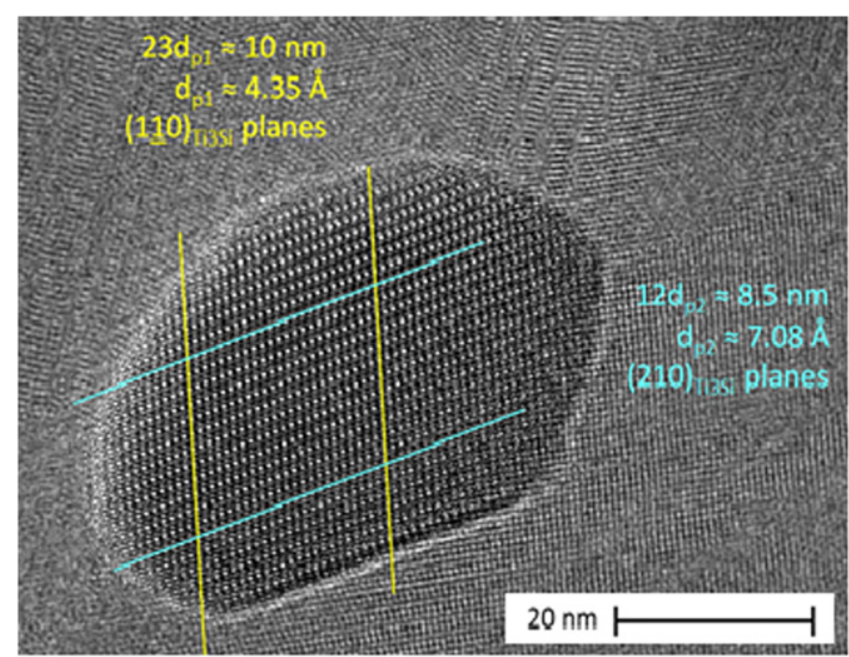

Figure 6. High resolution TEM image of a QD inside the polycrystalline sample. The plane distances correspond to the (110) and (210) planes of the $\mathrm{Ti}_{3} \mathrm{Si}$ tetragonal phase.

For the monocrystalline samples, the electrical resistivity values increase with the temperature. The main reason for this is the reduction of the charge carrier's mobility at higher temperatures due to the increase of carrier's scattering by phonons, which is coherent with the metallic characteristic of these high-doped semiconductors. For the polycrystalline samples, the electrical resistivity exhibits tiny changes with the temperature. In that case, the scattering on grain boundaries is the dominant scattering effect [4].

Moreover, as shown in table 1 , this effect is higher than any other scattering mode, so the observed mobility values are much lower (18 and $\left.13 \mathrm{~cm}^{2} \mathrm{~V}^{-1} \mathrm{~s}^{-1}\right)$ for the polycrystalline materials than for the monocrystalline materials (73 and $56 \mathrm{~cm}^{2} \mathrm{~V}^{-1} \mathrm{~s}^{-1}$ ). As grain boundaries can act as trapping sites for the carriers, their accumulation results in the creation of a potential energy barrier for the carrier transport [12]. By increasing the material's temperature, more energy is available for the carriers to cross the potential barrier, and the mobility increases.

Moreover, the values of the room-temperature electrical resistivity are considerably higher for the polycrystalline samples (around $1 \times 10^{-2} \Omega \mathrm{cm}$ ) compared to the monocrystalline samples (around $1 \times 10^{-3} \Omega \mathrm{cm}$ ), due to the reduction of the electronic carriers' mobility (see table 1 ).

A considerable increase in the Seebeck coefficient is observed for the QDSL samples. For the monocrystalline samples, the Seebeck coefficient is around $100 \mu \mathrm{V} \mathrm{K}^{-1}$ and $190 \mu \mathrm{V} \mathrm{K}^{-1}$ at $50{ }^{\circ} \mathrm{C}$ for the reference and the QDSL, respectively, resulting in a $90 \%$ increase with the inclusion of the QDs. For the polycrystalline samples, the Seebeck coefficient is around $130 \mu \mathrm{V} \mathrm{K}^{-1}$ and $230 \mu \mathrm{V} \mathrm{K}^{-1}$ at $50{ }^{\circ} \mathrm{C}$ for the reference and the QDSL, respectively, which is a $77 \%$ increase as a result of the inclusion of QDs.

The increase in the Seebeck coefficient, combined with a similar electrical resistivity, results in a large increase in the QDSLs' power factor, as shown in figures 9(b) and 10(b). These results clearly demonstrate the influence of the inclusion of Ti-based QDs on the power factor's improvement.

Moreover, the increase in the power factor is obtained here by an increase in the Seebeck coefficient and a stable electrical resistivity, which is the opposite of how it was obtained in the literature [9]. But as explained previously, our references samples have been voluntarily made with the same 


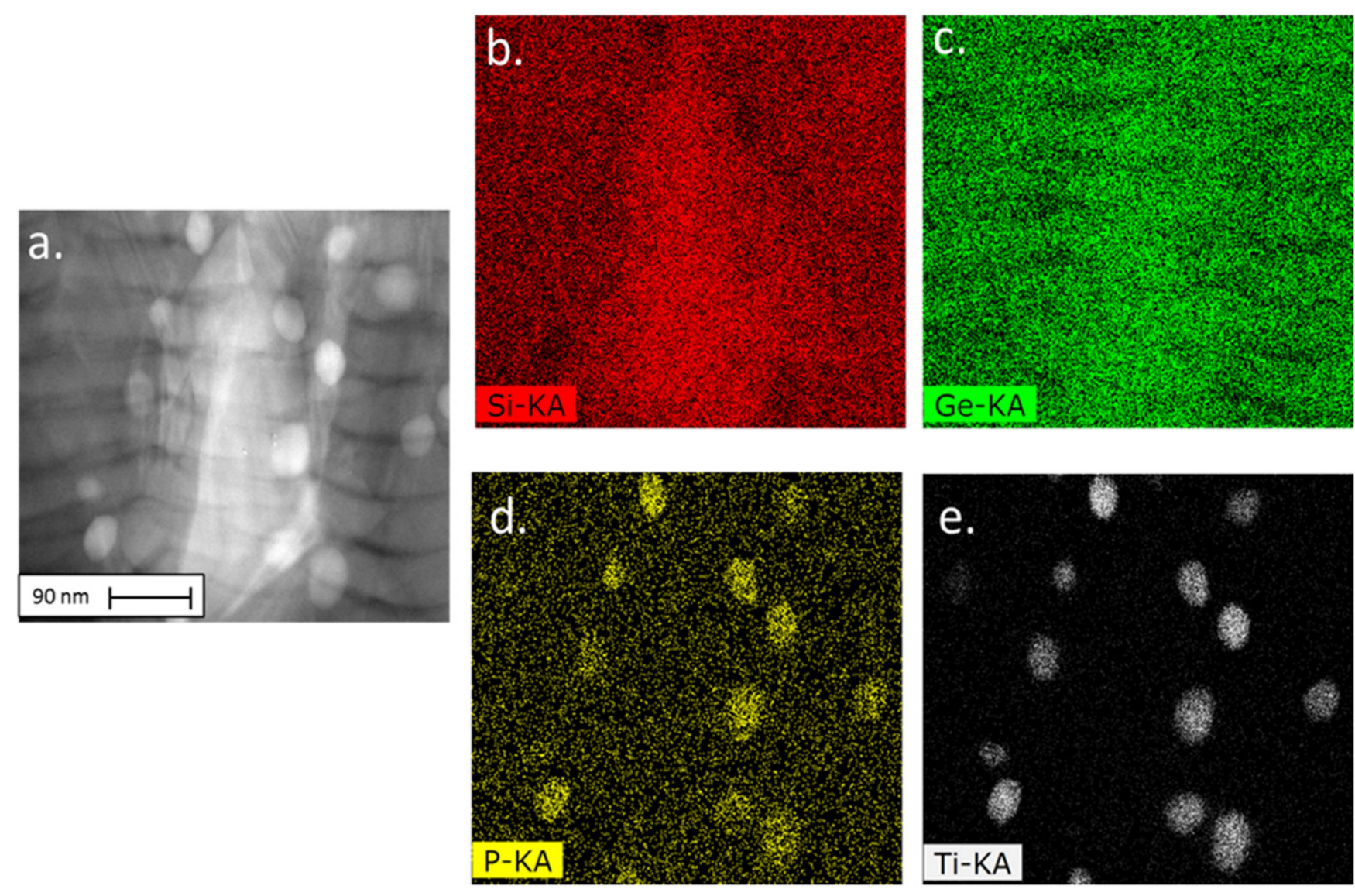

Figure 7. EDS chemical mapping of the polycrystalline sample: (a) STEM/HAADF image, (b) silicon signal, (c) germanium signal, (d) phosphorus signal, and (e) titanium signal. Note the accumulation of the phosphorus dopant in the QD zone.

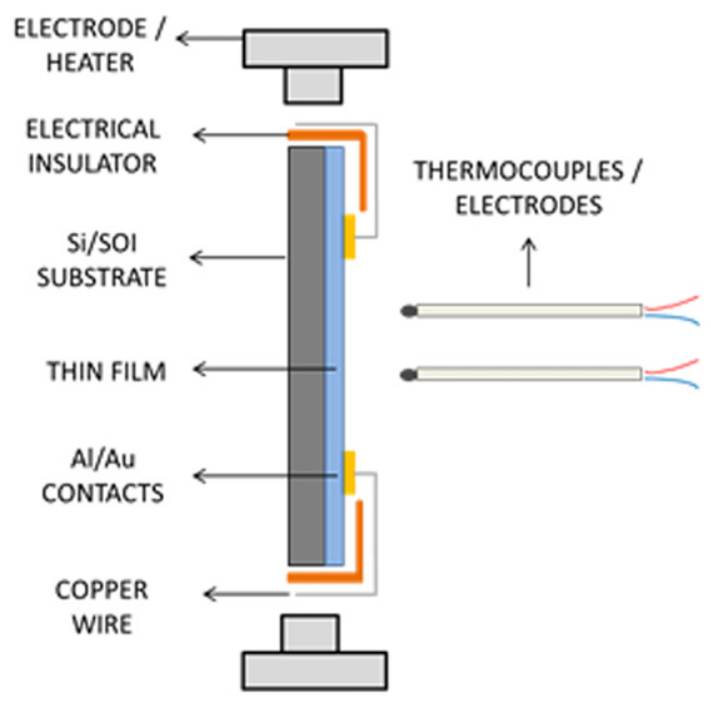

a.

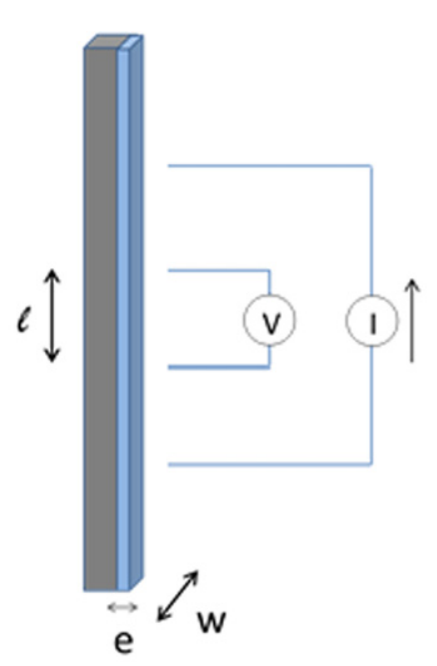

b.

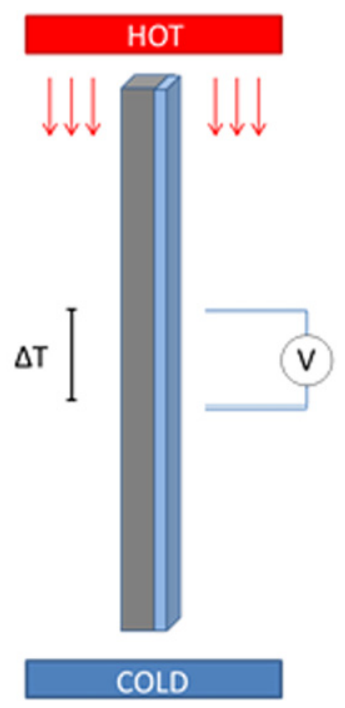

c.

Figure 8. Representation of the ZEM 3 measurement system, adapted to characterize the thin films. (a) Schematic view of the sample inside the system. (b) Representation of the electrical conductivity measurement. (c) Representation of the Seebeck coefficient measurement.

electrical conductivity as the QDSL to evaluate the effect of including QDs on the material's Seebeck coefficient. This prevents a strict comparison between these works.

Thermal conductivities obtained by TDTR are also presented in table 1. For the monocrystalline samples, a decrease in thermal conductivity is obtained for the QDSL $\left(6.8 \mathrm{~W} \mathrm{~m}^{-1} \mathrm{~K}^{-1}\right)$ compared to the reference $\left(8.5 \mathrm{~W} \mathrm{~m}^{-1} \mathrm{~K}^{-1}\right)$. This result shows the effect of nanostructuration by including silicide QDs in the reduction of the material's thermal conductivity. Reference values of $6.1 \mathrm{~W} \mathrm{~m}^{-1} \mathrm{~K}^{-1}$ for a 

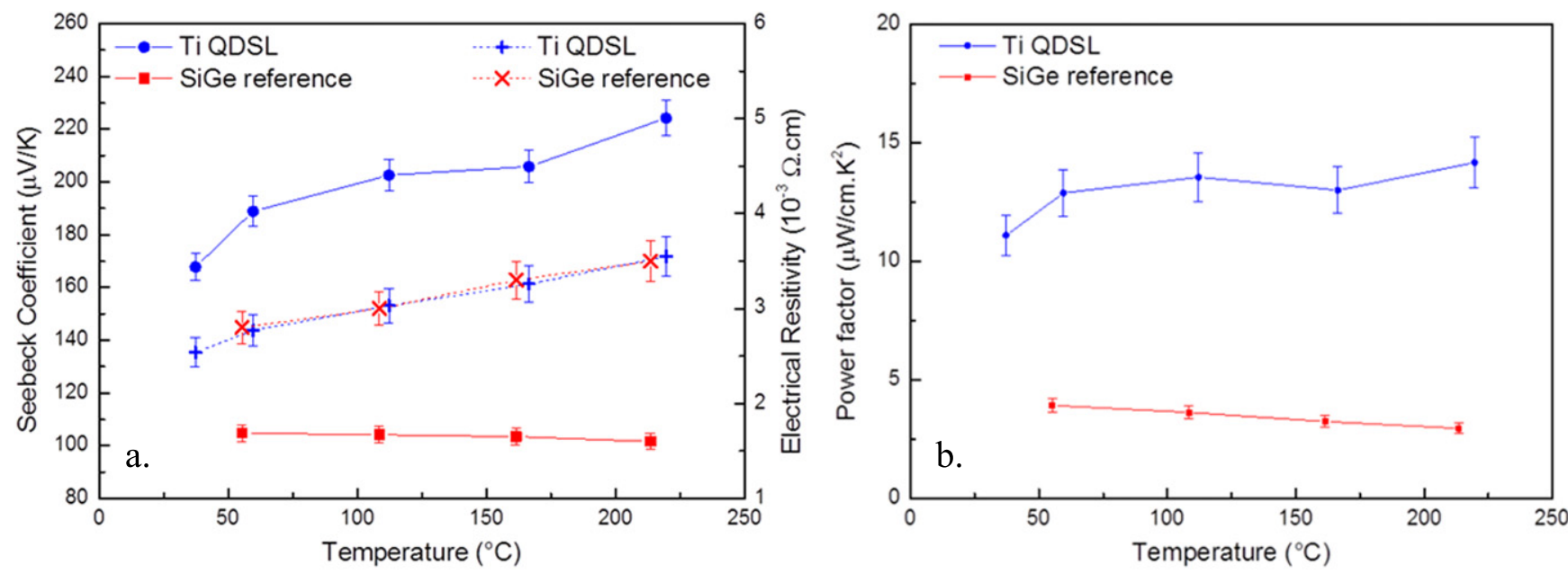

Figure 9. Seebeck coefficient, electrical resistivity (a), and power factor (b) of the monocrystalline QDSL (blue color) and the corresponding SiGe reference (red color) as a function of the temperature.
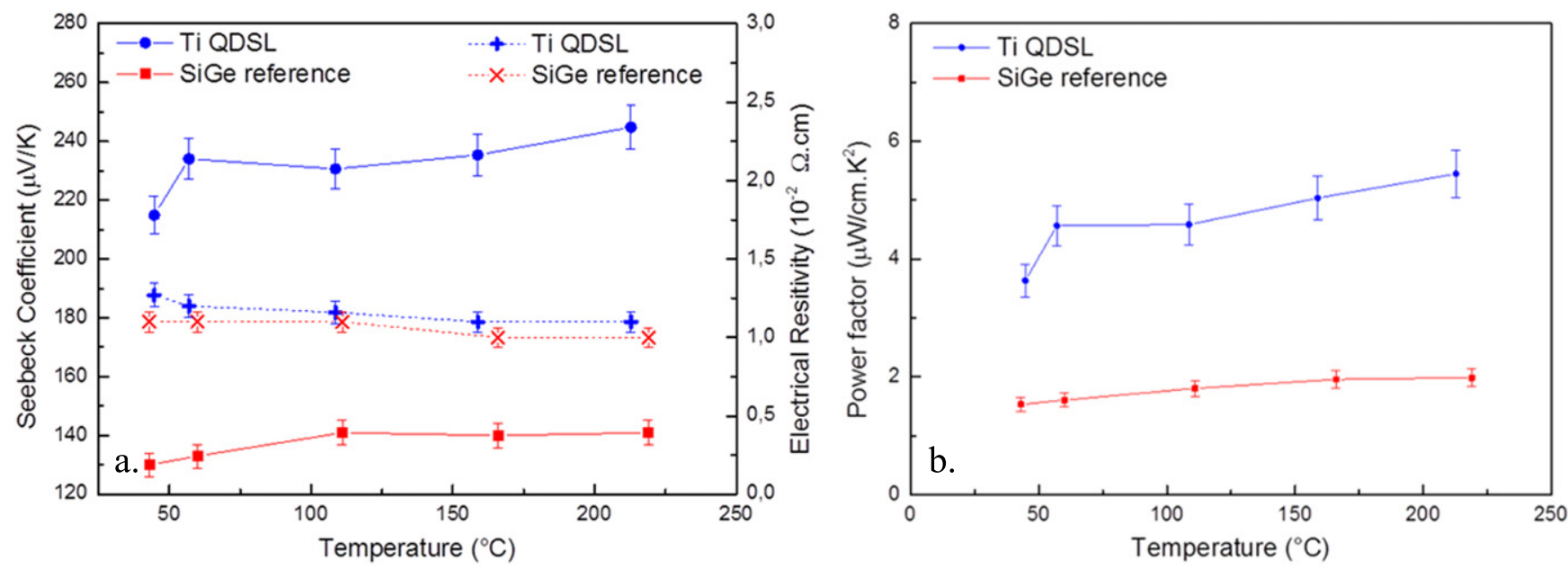

Figure 10. Seebeck coefficient, electrical resistivity (a), and power factor (b) of the polycrystalline QDSL (blue color) and the corresponding $\mathrm{SiGe}$ reference (red color) as a function of the temperature.

Table 1. Comparison of the carriers' concentration, $n$, mobility, $\mu$, and thermal conductivity, $\lambda$, between the monocrystalline and polycrystalline QDSLs and their corresponding SiGe references at room temperature.

\begin{tabular}{lcccccc}
\hline & \multicolumn{3}{c}{ Monocrystalline samples } & \multicolumn{3}{c}{ Polycrystalline samples } \\
\hline & $n$ & $\mu$ & $\lambda$ & $n$ & $\mu$ & $\lambda$ \\
& $\left(10^{19} \mathrm{~cm}^{-3}\right)$ & $\left(\mathrm{cm}^{2} \mathrm{~V}^{-1} \mathrm{~s}^{-1}\right)$ & $\left(\mathrm{W} \mathrm{m}^{-1} \mathrm{~K}^{-1}\right)$ & $\left(10^{19} \mathrm{~cm}^{-3}\right)$ & $\left(\mathrm{cm}^{2} \mathrm{~V}^{-1} \mathrm{~s}^{-1}\right)$ & $\left(\mathrm{W} \mathrm{m}^{-1} \mathrm{~K}^{-1}\right)$ \\
QDSL & $3.2 \pm 0.2$ & $73 \pm 5$ & $6.8 \pm 0.7$ & $2.8 \pm 0.2$ & $18 \pm 1$ & $4.6 \pm 0.5$ \\
Reference & $3.2 \pm 0.2$ & $56 \pm 3$ & $8.5 \pm 0.8$ & $4.4 \pm 0.2$ & $13 \pm 1$ & $4.2 \pm 0.4$ \\
\hline
\end{tabular}

monocrystalline $\mathrm{Si}_{0.85} \mathrm{Ge}_{0.15}$ thin film and about $8 \mathrm{~W} \mathrm{~m}^{-1} \mathrm{~K}^{-1}$ for a $\mathrm{Si}_{0.5} \mathrm{Ge}_{0.5}$ monocrystalline bulk sample are in good agreement with the values reported in the literature [4, 7].

The main difference in the behavior of the mono- and polycrystalline QDSLs can be seen in the thermal conductivity values. In the case of the polycrystalline samples, no change is observed when QDs are included inside the SiGe matrix because the grain boundaries are the dominant mechanism for phonon diffusion, masking the contribution of the phonons' diffusion with the QDs. The thermal conductivity values of the mono- and polycrystalline reference samples are 8.5 and $4.2 \mathrm{~W} \mathrm{~m}^{-1} \mathrm{~K}^{-1}$, respectively. By taking into account that both $\mathrm{SiGe}$ references have a aimilar Ge content and similar carrier concentration, the explanation of the grain boundary effect acting as the main and dominating diffusion mechanism is demonstrated.

As power factors have been measured in the in-plane direction while thermal conductivities have been measured in the cross-plane direction, it is not possible to calculate the ZT values of these anisotropic QDSLs. Nevertheless, a significant 
improvement in both the power factors and thermal conductivities has been demonstrated, even in different directions, proving the beneficial influence of nanostructuration on the thermoelectric properties.

\section{Conclusions}

We present here the first instance of the growth of n-doped monocrystalline and polycrystalline QDSLs using the CVD technique. TEM analyses have confirmed the presence of Ti-based silicide inclusions inside the matrix, and have shown the specific behavior of the QDs during the growth, with a coalescence phenomenon, for the polycrystalline QDSLs.

Additionally, both the mono- and polycrystalline QDSLs exhibit a significant increase in their power factors after the inclusion of the QDs up to four times higher for the monocrystalline sample compared to the SiGe reference, and 2.3 times higher for the polycrystalline sample. Finally, the monocrystalline QDSLs also present a $20 \%$ reduction in their thermal conductivity due to the inclusion of QDs.

These results show that a great improvement of the TE materials' properties is obtained by including QDs in an SiGe matrix, making these materials very promising for energyharvesting or cooling applications.

\section{References}

[1] Böttner H, Nurnus J, Gavrikov A, Kühner G, Jägle M, Künzel C, Eberhard D, Plescher G, Schubert A and Schlereth K H 2004 J. Microelectromech. Syst. 13414

[2] Harman T C, Taylor P J, Walsh M P and LaForge B E 2002 Science 2972229

[3] Hicks L D and Dresselhaus M S 1993 Phys. Rev. B 4712727

[4] Savelli G, Plissonnier M, Remondière $\mathrm{V}$, Bablet $\mathrm{J}$ and Fournier J M 2008 J. Micromech. Microeng. 18104002

[5] Ziouche K, Savelli G, Bougrioua Z, Hauser D, Lejeune P, Michon P M, Lasri T and Leclercq D 2014 J. Appl. Phys. 116043701

[6] Pernot G et al 2010 Nat. Mater. 9491

[7] Mingo N, Hauser D, Kobayashi N P, Plissonnier M and Shakouri A 2009 Nano Lett. 9711

[8] Favier K, Bernard Granger G, Navone C, Soulier M, Boidot M, Leforestier J, Simon J, Tedenac J C and Ravot D 2014 Acta Mater. 64429

[9] Yu B, Zebarjadi M, Wang H, Lukas K, Wang H, Wang D, Opeil C, Dresselhaus M, Chen G and Ren Z 2012 Nano Lett. 122077

[10] Dilhaire S, Rampnoux J M, Grauby S, Pernot G and Calbris G 2009 ASME Proc. p 451

[11] Pernot G, Michel H, Vermeersch B, Burke P, Lu H, Rampnoux J M, Dilhaire S, Ezzahri Y, Gossard A and Shakouri A 2011 MRS Online Proc. Libr. p 1347

[12] Seto J Y W 1975 J. Appl. Phys. 465247

[13] Savelli G, Hauser D, Michel H and Simon J 2012 AIP Conf. Proc. 1449275

[14] Hauser D, Savelli G, Plissonnier M, Montès L and Simon J 2012 Thin Solid Films 5204259 